\title{
Ab initio calculations of the cohesive energy and the bulk modulus of Aluminium
}

\author{
R. Gaudoin and W. M. C. Foulkes \\ CMTH Group, Blackett Laboratory, Imperial College of Science, \\ Technology and Medicine, Prince Consort Road, London SWr 2BW, England \\ G. Rajagopal \\ TCM Group, Cavendish Laboratory, Cambridge University, Madingley Road, Cambridge CB3 OHE
}

(Dated: April 18, 2008)

\begin{abstract}
To date there have been few attempts to calculate bulk properties such as the cohesive energy or the bulk modulus of metals using Monte Carlo methods. We present a variational Monte Carlo calculation of aluminium and find that methods used to deal with finite-size effects work just as well as in insulators, despite the presence of a Fermi surface. However, the large statistical uncertainties are a problem when evaluating the bulk modulus.
\end{abstract}

PACS numbers: PACS: 71.15.Nc, 62.20.-x, 02.70.Uu

\section{INTRODUCTION}

Two common methods used for numerical ab initio calculations of the bulk modulus, lattice constant and cohesive energy of solids are density functional theory (DFT), often within the local density approximation (LDA), and Monte Carlo (MC) calculations such as variational (VMC) and diffusion (DMC) Monte Carlo. The latter are usually based on DFT Kohn-Sham orbitals. To date, there have been few published MC studies of metals (see for example Eckstein et al. ${ }^{1}$ and Yao et $a .^{2}{ }^{2}$ ). In this paper we present LDA and VMC calculations of bulk FCC aluminium, the aim being to test the applicability to metals of standard methods used to tackle the unavoidable finite-size errors in MC simulations.

It has been suggested that, for metals, MC methods might be inadequate, due to the computational difficulty of dealing with large simulation cells (equivalent to dense $\mathrm{k}$-point grids in DFT), which one might think are necessary in order to sample the partially filled bands of a metal accurately. Reassuringly, however, we find that VMC is no less accurate for aluminium than for some typical non-metallic substances. Unfortunately, as is also the case for insulators, statistical noise causes major problems when evaluating the bulk modulus.

This paper is organised as follows. Sec. II gives a brief recap of some bulk properties and their measured values. Computational issues are discussed in Sec. III, in Sec. IV we present our results and Sec. V concludes the paper.

\section{THE BULK MODULUS, THE LATTICE PARAMETER AND THE COHESIVE ENERGY}

\section{A. Definitions}

For an FCC lattice and a given equation of state (EOS), $E$ as a function of lattice parameter $a$ or volume
$V=\frac{1}{4} a^{3}$, the bulk modulus $B$ is defined by the equation

$$
B=\left(V \frac{\partial^{2} E}{\partial V^{2}}\right)=\frac{4}{9 a} \frac{\partial^{2} E}{\partial a^{2}}
$$

evaluated at the minimum.

The position of the minimum of the EOS defines the equilibrium lattice parameter and unit cell volume at zero pressure. The cohesive energy is then the difference between the energy per atom of the bulk material at equilibrium and the energy of a free atom in its ground state.

\section{B. Experimental data}

Electronic structure calculations yield the energy of a system where the nuclei are frozen point-like particles. In reality, however, the nuclei, like electrons, are quantum mechanical objects. The effects of quantum mechanics on the nuclei are normally quite small, but need to be taken into account, at least approximately, when accurate results are required. Recently, such calculations have been performed within the quasi-harmonic approximation (see Xie et $a l .^{3,4}$ ), which necessitates the evaluation of the entire phonon spectrum. This is a rather complex task and is currently impossible within a MC context owing to the fact that effective MC system sizes cannot be made bigger than a few unit cells using today's computers. This limitation of the system size limits the wavelengths of the phonons that can be studied. An alternative method is to use the Debye temperature, $\Theta_{D}$, to estimate the phonon contribution. We can then derive adjusted experimental values which are directly comparable to standard electronic structure calculations.

In the case of aluminium, the energy per atom, $\zeta_{z}$, of the phonon ground state can be estimated within the Debye theory: $\zeta_{\mathrm{z}}=\frac{9}{8} k_{B} \Theta_{D}$. If we subtract this value from the experimental value we get a number directly comparable to results from standard electronic structure calculations. We take the exact zero-temperature cohesive energy to be $3.39 \mathrm{eV}$, as given by Kittel, ${ }^{5}$ and adjust 
it using a value ${ }^{6}$ of $\Theta_{D}=428^{\circ} \mathrm{K}$. A similar approach ${ }^{7,8}$ yields comparable values for the bulk modulus and the lattice parameter. Table I shows the unadjusted and adjusted experimental data.

\section{COMPUTATIONAL IMPLEMENTATION}

\section{A. The atomic LDA calculation and the pseudopotential}

A code written by Fuchs et al. ${ }^{9}$ was used to carry out the full-core atomic density-functional calculation within the local spin density approximation (LSDA) ${ }^{10}$ and included relativistic effects within a scalar-relativistic approximation. ${ }^{9,11}$ The Kohn-Sham equation was solved iteratively on a logarithmic radial mesh defined by the starting value $4.80769 \times 10^{-4}$ Hartree atomic units (a.u.) and the scale factor 1.0247, using 493 grid points giving a cutoff distance of about 80 a.u.

As it is not practical to compute the energy of a solid of full-core 13-electron atoms, we used a non-local norm-conserving pseudopotential based on the atomic orbitals with angular momentum components $l=0,1,2$. The use of pseudopotentials improves computational efficiency and, in addition, reduces the signal to noise ratio in the MC calculation. We used the Hamann ${ }^{12}$ algorithm for the generation of the pseudopotential.

\section{B. The bulk LDA calculation}

Since we used the bulk LDA calculations as an input for the VMC study, it was important to choose a $k$-point sampling mesh that was consistent with the MC simulation cell. Moreover, as we wanted to build a real trial wave function from the orbitals, we had to use a grid with an offset from the origin of half a reciprocal lattice vector. The choice of this offset can strongly affect the convergence rates of both the LDA and VMC energies as functions of the size of the simulation cell. Work on insulators by Rajagopal et al. ${ }^{13}$ has found that particularly good results are obtained when the k-point offset, expressed in terms of the basis vectors of the reciprocal lattice, is equal to $(0.5,0.5,0.5)$. Our investigations have confirmed that this is also a good choice for aluminium. The k-point grids we used are closely related to those introduced by Monkhorst and Pack. ${ }^{13-15}$

To perform these LDA calculations we used the code of Boeckstedte et al., ${ }^{16}$ where the plane-wave cut-off was 20 Rydbergs and the parametrisation of the LDA was that obtained by Perdew and Zunger. ${ }^{17}$ The grid corresponded to a $10 \times 10 \times 10$ FCC superlattice.

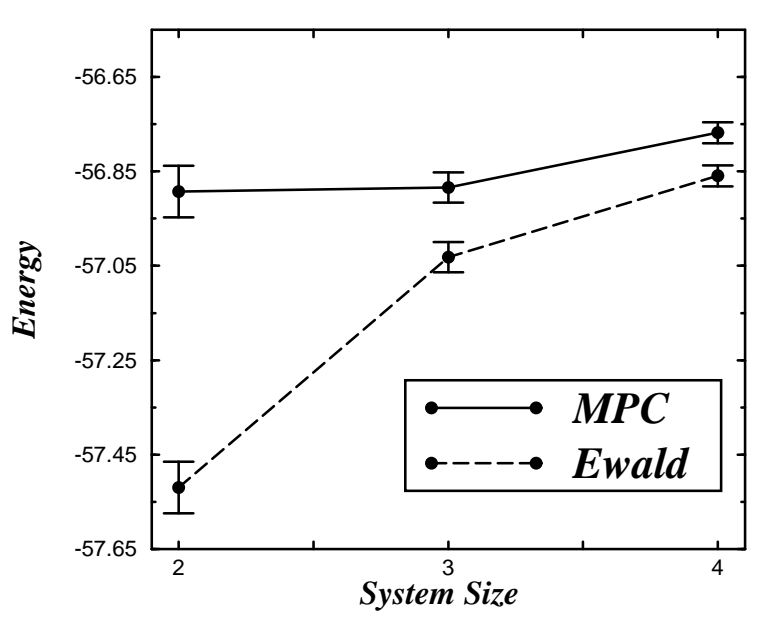

FIG. 1: This figure shows the convergence of the energy (eV per unit cell) as the system size is increased from $a 2 \times 2 \times 2$ to $a 4 \times 4 \times 4$ super cell geometry. The data has been corrected for a possible LDA trend and both results using standard Ewald and the MPC interactions are shown.

\section{The VMC calculation}

As an input to the VMC calculations we used the LDA orbitals of the pseudo-atom and pseudo-solid respectively. The VMC trial wave function was based on a homogeneous Jastrow factor of the form described in Williamson et al. ${ }^{18}$ with parametrised one- and twobody terms with 16 parameters each, which we converged using several variance minimisation runs. Depending on the system size, the MC calculations used between $10^{4}$ and $10^{5}$ configurations of all the electrons.

To reduce finite-size effects we used the model periodic coulomb (MPC) interaction ${ }^{19,20}$ (see also Fig. (1)). In addition, we estimated the orbital intrinsic finite-size effects by looking at the trends in the LDA energies as the system size was increased. For example, from the VMC energy for the $3 \times 3 \times 3$ system, we subtracted the difference in LDA energy between the $3 \times 3 \times 3$ and $10 \times 10 \times 10$ systems, tacitly assuming that the latter was sufficiently converged. Fig. (1) gives the trends of the VMC energy for increasing system size. Note that by construction MPC and Ewald data agree for infinite systems. In fact, for the $4 \times 4 \times 4$ system, they differ by between one and two standard deviations only. This, plus the reasonable assumption of monotonic convergence, and the fact that the more reliable MPC data changes insignificantly anyway, suggests that the MPC result for the $4 \times 4 \times 4$ system is sufficiently converged.

One important question regarding MC calculations of metals is what to do about partially filled orbitals. A typical structure of the Kohn-Sham energies at various $\mathrm{k}$-points in a $2 \times 2 \times 2$ system is shown in Fig. 2. This sys- 


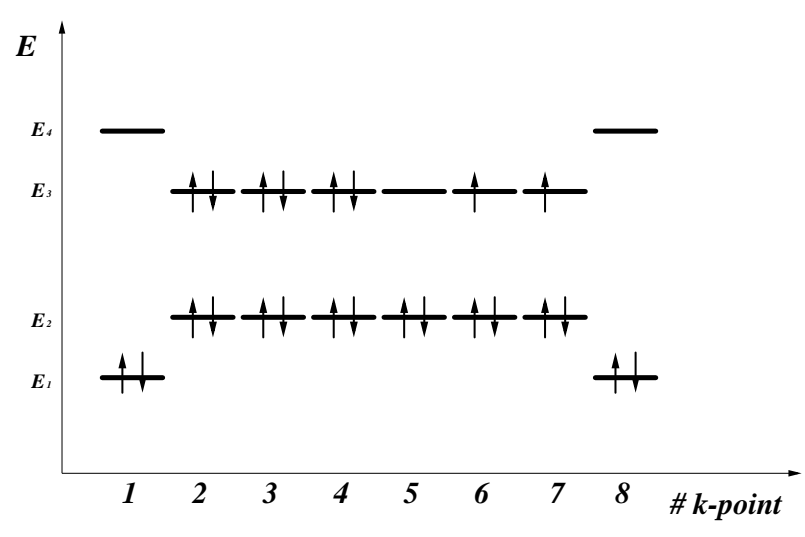

FIG. 2: This figure shows the Kohn-Sham energies at the 8 $k$-points that correspond to $a 2 \times 2 \times 2$ system. The degeneracies are due to symmetry and each orbital can at most hold 2 electrons indicated by the up- and down-arrows. The distribution of the electrons shown here is a system intermediate between a fully polarised and an unpolarised system. The energies are $E_{1}=-3.996 \mathrm{eV}, E_{2}=0.439 \mathrm{eV}, E_{3}=4.749 \mathrm{eV}$, and $E_{4}=8.961 \mathrm{eV}$.

tem contains 8 three electron pseudo atoms, making 24 electrons in total. We see that in the ground state there are 12 orbitals at $E_{3}$ but only 8 electrons to fill these. In DFT this discrepancy is overcome by including a factor of $\frac{2}{3}$ whenever the orbitals at $E_{3}$ are used to calculate the energy or density. In contrast, $\mathrm{MC}$ orbitals have to be fully occupied or empty. When dealing with jellium, technically also a metal, the problem of partially occupied orbitals is "solved" by choosing the system size carefully such that for a given density all degenerate electron levels are occupied ("filling a star"). Effectively, such a jellium system is then no longer a metal; it becomes a semiconductor with a small gap. In real solids, however, where the geometry and electron density are given, this procedure is no longer applicable, and we have to compare different occupation schemes, one of which is indicated by the up- and down-arrows in Fig. 2. We compared several different occupation schemes (unpolarised, fully polarised and an intermediate system) for the degenerate one-electron orbitals at the Fermi level of the $2 \times 2 \times 2$ system and found, reassuringly, that a spinunpolarised trial wave function gives the lowest VMC energy for bulk aluminium. The converged VMC energies are $-57.29(5) \mathrm{eV}$ for the spin unpolarised system and $-57.26(6) \mathrm{eV}$ and -57.20 .(6)eV for the fully polarised and the intermediate system respectively.

\section{RESULTS}

The results given in this section are compared to experimental data from which the effects of finite temperature and zero-point motion have been subtracted. ${ }^{7}$
TABLE I: This table shows the experimental values for the cohesive energy $E^{c}$ (eV/atom), the equilibrium bulk modulus $B$ $\left(10^{11} \mathrm{Nm}^{-2}\right)$, its pressure derivative $B^{\prime}=\frac{\partial B}{\partial P}$ (dimensionless) and the equilibrium lattice parameter a $(\AA)$, for bulk aluminium. The values adjusted for the effect of zero-point motion and finite temperature effects are also given $\left(E_{0}^{c}, B_{0}\right.$, and $\left.a_{0}\right)$. The $L D A$ and $V M C$ values of $E_{0}^{c}, B_{0}$, and $a_{0}$ were estimated using a quartic fit to the calculated equation of state $E(a)$. The LDA bulk modulus is very close to the adjusted value.

\begin{tabular}{cccccccc} 
& $E^{c}$ & $B$ & $B^{\prime}$ & $a$ & $E_{0}^{c}$ & $B_{0}$ & $a_{0}$ \\
\hline EXP & 3.39 & 0.759 & 4.267 & 4.05 & 3.43 & 0.813 & 4.022 \\
LDA & - & - & 4.83 & - & 4.21 & 0.802 & 3.960 \\
VMC & - & - & $6.9(1.1)$ & - & $3.23(8)$ & $0.65(17)$ & $3.970(14)$
\end{tabular}

\section{A. Cohesive energy}

The cohesive energy within LDA is about $24 \%$ too large (see Table I), whereas the VMC cohesive energy for aluminium understates the experimental value by $5.8 \%$. This error is two and a half standard deviations and is thus statistically significant, but the VMC result is clearly much better than the LDA result. The cohesive energy was calculated at the experimentally determined lattice parameter.

\section{B. Bulk modulus and lattice parameter}

We took the experimentally determined FCC crystal structure as an input and calculated the total energy for 14 different values of the lattice parameter ranging from 0.91 to 1.06 times the equilibrium value. This enabled us to determine the minimum of the $E(V)$ curve and hence the ground-state energy. When calculating the bulk modulus and lattice parameter, some care has to be taken to ensure the fitting is done correctly. It is important to use a sufficiently flexible functional form for the EOS. In addition, in the case of $\mathrm{VMC}$ data, we have to deal with statistical noise. Following our previous work ${ }^{7}$ we chose a parametrised quartic polynomial in $a$ as the generic form of the EOS. The raw data and the fitted curves are shown in Fig. 3 and the values of the bulk modulus and the lattice parameter are given in Table I.

The LDA lattice constant is slightly too small, although the bulk modulus is very accurate. This is surprising as most authors find that the LDA yields too large a bulk modulus. The better agreement obtained here is due to the finite-temperature and zero-point corrections, which raise the "ideal" calculated bulk modulus of aluminium by $7 \%$ relative to the experimental value.

Can we use VMC to improve on these LDA results, as we did for the cohesive energy? One obvious problem, which turns out to be serious, is that, unlike the LDA energies, the VMC energies are noisy. 


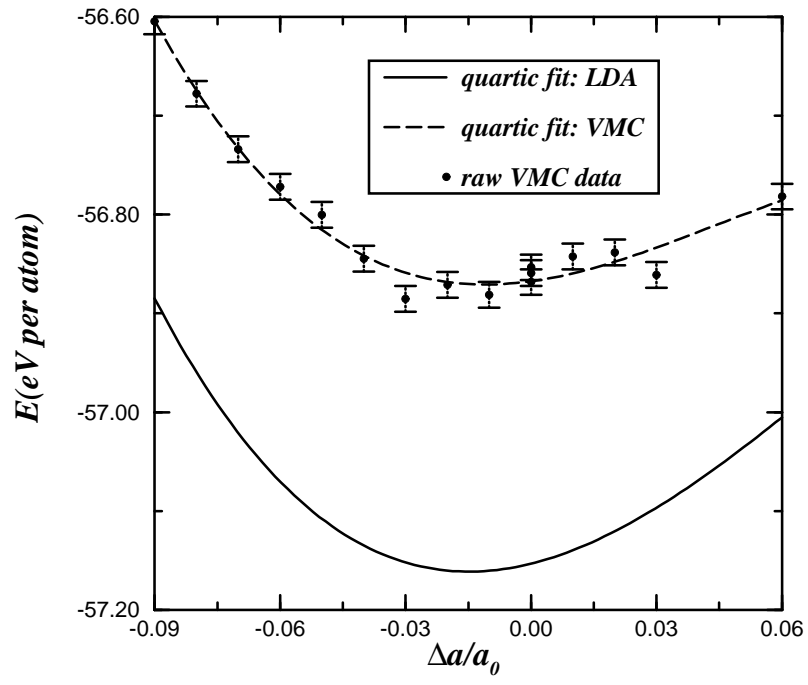

FIG. 3: This figure shows LDA and VMC data for E(a) fitted to a quartic polynomial. Raw VMC data including error bars are also given. Note that on the resolution of this figure the raw LDA data (not shown, evaluated at the same points as the $V M C$ data) is in fact indistinguishable from the fitted curve. Also, of $16 \mathrm{VMC}$ data points ( $\Delta a=0$ has been evaluated three times), 4 or 5 are more than one standard deviation removed from the fitted curve, implying that the fit is consistent with the VMC errors.

Looking at Table I, we see that the calculated lattice parameter is slightly more accurate than the LDA value, but the calculated bulk modulus appears somewhat worse. This "poor" results for the bulk modulus is, however, within a standard deviation of the ideal value. In contrast to the VMC bulk modulus, which is underestimated, $B^{\prime}$ is overestimated. This is consistent as the true VMC EOS is unlikely to differ considerably from the DFT EOS. If the estimated VMC curvature at the minimum is much smaller than its DFT counterpart, the VMC curvature has to change more rapidly than for DFT. Since the curvature is the main ingredient of the bulk modulus, the same will hold for $B^{\prime}$.

We regard it as unlikely that the VMC method actually gives worse results than the LDA, especially as the LDA orbitals are used as input to the VMC calculations. We believe, therefore, that a reduction of the statistical error, e.g, by using correlated sampling, ${ }^{21}$ is necessary to obtain more accurate VMC results.

\section{CONCLUSIONS}

The presence of a Fermi surface, and the inability to sample it well in MC simulations with small simulation cells (corresponding to coarse Meshes of $\mathrm{k}$ points), do not limit the applicability of standard MC methods in the studies of metals. This might seem surprising at first. However, one must not forget that we apply to the MC results a DFT-based finite-size correction that takes account of the change in DFT total energy as the density of the DFT k-point sampling mesh is increased. This deals with the one-body contributions to the MC finitesize error. The MC method treats the many-body correlation energy explicitly in real space by modelling the exchange-correlation hole using a Jastrow factor. Since the hole is fairly short ranged, we expect the exchangecorrelation energy to converge rapidly as the size of the simulation cell is increased. The many-body finite-size error is therefore small. The largest remaining finite-size error arises from spurious interactions between the periodically repeated copies of the exchange-correlation hole. This error is adequately dealt with using the MPC interaction. Summarising, we belileve that the main effect of the Fermi surface on the MC energy

There is the usual limitation due to the statistical nature of MC calculations. Since energy derivatives such as the bulk modulus are very susceptible to noise in the data, the statistical nature of the MC method makes accurate MC calculations of bulk moduli difficult. This limitation applies in metallic and insulating systems.

In contrast to much previous work, we find that the LDA produces a very accurate value for the bulk modulus of Al. The accuracy of our result can be attributed to the inclusion of finite-temperature and zero-point motion effects. For aluminium, these effects imply that the calculated bulk modulus ought to be approximately $7 \%$ greater than the measured value, explaining the apparent over-estimation of the bulk modulus obtained by other authors. $^{22,23}$
${ }^{1}$ H. Eckstein, and W. Schattke, Physica A 216, 151 (1995).

${ }^{2}$ G. Yao, J. G. Xu, and X. W. Wang, Phys. Rev. B 54, 8393 (1996).

3 J. Xie, S. P. Chen, J, S. Tse, S. de Gironcoli, and S. Baroni, Phys. Rev. B 60, 9444 (1999).

4 J. Xie, S. P. Chen, H. V. Brand, and R. L Rabie, J. Phys.: Cond. Mat. 12, 8953 (2000).

${ }^{5}$ C. Kittel, Introduction to Solid State Physics, sixth edition, John Wiley, (1986).

${ }^{6}$ American Institute of Physics handbook, 3rd ed., edited by
E. G. Dwight and B. H. Billings, (American Institute of Physics, 1972).

7 R. Gaudoin, and W. M. C. Foulkes, accepted for publication in Phys. Rev. B (Brief report, 2002).

8 M. M. Dacorogna and M. L. Cohen, Phys. Rev. B 34, 4996 (1986).

9 M. Fuchs, M. Scheffler, Comput. Phys. Commun. 119, 67 (1999).

10 J. A. Perdew, Y. Wang, Phys. Rev. B 45, 13244 (1992).

11 D. D. Koelling, B. N. Harmon, J.Phys. C 10, 3107 (1977). 
${ }^{12}$ D. R. Hamann, Rhys. Rev. B 40, 2980 (1989).

13 G. Rajagopal, R. J. Needs, A. James, S. D. Kenny, and W. M. C. Foulkes, Phys. Rev. B 51, 10591 (1995).

14 H. J. Monkhorst, and J. D. Pack, Phys. Rev. B 13, 5188 (1976).

15 G. Rajagopal, R. J. Needs, S. D. Kenny, W. M. C. Foulkes, and A. James, Phys. Rev. Lett. 73, 1959 (1994).

16 M. Boeckstedte, A. K. Kley, J. Neugebauer, M. Scheffler, Comput. Phys. Commun. 107, 187 (1997).

17 J. P. Perdew, and A. Zunger, Phys. Rev. B 23, 5048 (1981).

18 A. J. Williamson, S. D. Kenny, G. Rajagopal, A. J. James, R. J. Needs, L. M. Fraser, W. M. C. Foulkes, and P. Maccallum, Phys. Rev. B 53, 9640 (1996).
19 L. M. Fraser, W. M. C. Foulkes, G. Rajagopal, R. J. Needs, S. D. Kenny, and A. J. Williamson, Phys. Rev. B 53, 1814 (1996).

20 A. J. Williamson, G. Rajagopal, R. J. Needs, L. M. Fraser, W. M. C. Foulkes, Y. Wang, and M.-Y. Chou, Phys. Rev. B 55, R4851 (1997).

${ }^{21}$ C. J. Umrigar, Int. J. Quantum Chem. 23, 217 (1989).

${ }^{22}$ R. Zeller, M. Asato, T. Hoshino, J. Zabloudil, P. Weinberger, and P. H. Dederichs, Phil. Mag. B 78, 417 (1998).

23 A. Khein, D. J. Singh, C. J. Umrigar, Phys. Rev. B 51, 4105 (1995). 